\title{
Theoretical studies of oscillatory behavior for long-range exchange bias
}

\author{
Yuan-Jen Lee ${ }^{\mathrm{a}, *}$, Ching-Ray Chang ${ }^{\mathrm{a}}$, Tzay-Ming Hong ${ }^{\mathrm{b}}$, C.H. Ho ${ }^{\mathrm{a}}$, \\ Minn-Tsong Lin ${ }^{\mathrm{a}}$ \\ ${ }^{a}$ Department of Physics, National Taiwan University, 1. Sec. 4 Roosevelt Rd., Taipei, Taiwan \\ ${ }^{\mathrm{b}}$ Department of Physics, National Tsing Hua University, Hsinchu, Taiwan
}

\begin{abstract}
We have calculated the long-range exchange bias between a ferromagnet and an antiferromagnet separated by a nonmagnetic metal spacer. The RKKY interaction and dipolar interaction are used to analyze the temperature effects and thickness dependence in trilayer structure. The experimental observed oscillatory exchange bias through the metal spacer are in good agreement with the calculated results. (C) 2002 Elsevier Science B.V. All rights reserved.
\end{abstract}

Keywords: Exchange bias; Exchange coupling - RKKY; Dipolar interactions

More than 40 years ago, the hysteresis loop displacement was first observed in the antiferromagnetic coated magnetic particles by Meiklejohn and Bean [1,2], and it was known as the exchange bias $\left(H_{\mathrm{E}}\right)$. In general, the origin of the $H_{\mathrm{E}}$ is the interaction between antiferromagnet (AFM) and ferromagnet (FM) layers at interfaces. On the other side, the long-range magnetic coupling between two FM separated by a nonmagnetic metal (M) spacer is discussed a lot both in the theories and experiments in the past decade [3-7]. As the thickness of the metal spacer is varied, the interlayer coupling between two FM layers oscillates, which alternates between FM and AFM coupling, extending to several tens of $\AA$. The oscillatory behavior can be explained successfully with RKKY-like models [6,7]. In 1997 Gökemeijer et al. [8] started to study the FM/M/ AFM trilayer system [8], in which the $H_{\mathrm{E}}$ can also be observed. However, the strength of the exchange coupling decays exponentially with increasing the thickness of the metal spacer, instead of oscillatory coupling like $\mathrm{FM} / \mathrm{M} / \mathrm{FM}$ trilayer system. Recently, some experiments reported that the oscillation of the long-range exchange bias was observed [9-11], e.g., in $\mathrm{NiFe} / \mathrm{Cu} / \mathrm{NiO}$ structures. Fig. 1 shows the $H_{\mathrm{E}}$ in

\footnotetext{
*Corresponding author. Fax: + 886-2-33665167.

E-mail address: b0326abc@ms26.hinet.net (Y.-J. Lee).
}

$\mathrm{NiFe}(100 \AA) / \mathrm{Cu} / \mathrm{NiO}(100 \AA)$ as a function of $\mathrm{Cu}$ thickness at different measured temperature. One finds that the $H_{\mathrm{E}}$ oscillates with $\mathrm{Cu}$ thickness, and this oscillatory tendency becomes significant with increasing temperature [11].

Even though the oscillatory behaves of $H_{\mathrm{E}}$ was attributed to the competition between RKKY and dipolar interaction, but the detailed analysis was lacking. Here, we theoretically analyze this oscillation of the $H_{\mathrm{E}}$ in $\mathrm{NiFe}(100 \AA) / \mathrm{Cu} / \mathrm{NiO}(100 \AA)$ in details. In our model, the interfacial spin in the AFM layer is assumed to be uncompensated, consequently there is a net magnetic moment $\left(\vec{m}_{\mathrm{AF}}\right)$ in the AFM at interfaces. Since the $\vec{m}_{\mathrm{AF}}$ couples strongly with neighbor spin within the AFM layer, and thus the $\vec{m}_{\mathrm{AF}}$ remains fixed when the magnetization $\left(\overrightarrow{\mathrm{M}}_{\mathrm{F}}\right)$ in the $\mathrm{FM}$ layer rotates coherently. For simplicity, we assumed that the FM anisotropy axis, AFM anisotropy axis, and the applied field are in the same direction, but the $\vec{m}_{\mathrm{AF}}$ and the cooling field are along the opposite direction. The energy per unit area in FM/M/AFM system can then be written as

$$
\begin{aligned}
\varepsilon(t)= & K_{\mathrm{F}} t_{\mathrm{F}} \sin ^{2} \theta-H M_{\mathrm{F}} t_{\mathrm{F}} \cos \theta \\
& +\varepsilon_{\mathrm{dip}}(t)+\varepsilon_{\mathrm{RKKY}}(t),
\end{aligned}
$$

where $t$ is thickness of $\mathrm{Cu}, K_{\mathrm{F}}$ is the anisotropy of the FM layer, $t_{\mathrm{F}}$ is the thickness of the FM layer, $H$ is the 


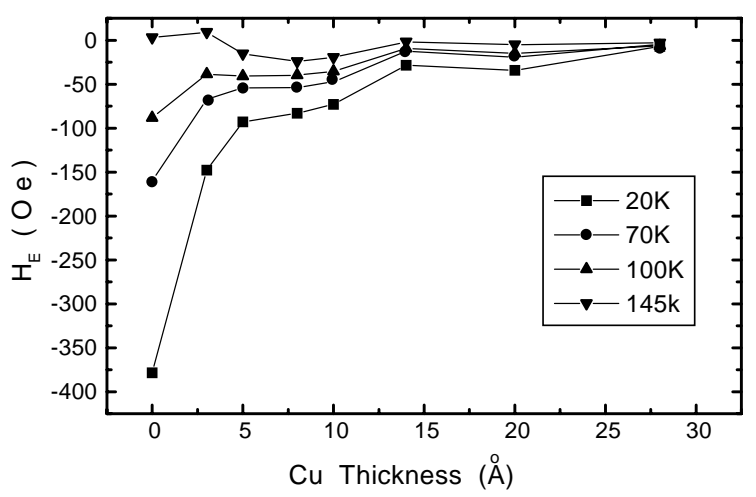

Fig. 1. The $H_{\mathrm{E}}$ values in $\mathrm{NiFe}(100 \AA) / \mathrm{Cu} / \mathrm{NiO}(100 \AA)$ as a function of $\mathrm{Cu}$ thickness at different measured temperature of $20,70,100$, and $145 \mathrm{~K}$.

applied field, and $\theta$ is the angle between $\overrightarrow{\mathrm{M}}_{\mathrm{F}}$ and anisotropy axis.

$$
\begin{aligned}
\varepsilon_{\mathrm{dip}}(t) & =\frac{\overrightarrow{\mathrm{M}}_{\mathrm{F}} \cdot \vec{m}_{\mathrm{AF}}-3\left(\overrightarrow{\mathrm{M}}_{\mathrm{F}} \cdot \hat{n}\right)\left(\vec{m}_{\mathrm{AF}} \cdot \hat{n}\right)}{t^{3}} t_{\mathrm{F}} \\
& =-\frac{1}{t^{3}} M_{\mathrm{F}} m_{\mathrm{AF}} t_{\mathrm{F}} \cos \theta
\end{aligned}
$$

is the dipole-dipole interaction between the $\overrightarrow{\mathrm{M}}_{\mathrm{F}}$ and $\vec{m}_{\mathrm{AF}}$, and

$$
\begin{aligned}
\varepsilon_{\mathrm{RKKY}}(t)= & \frac{4 J^{2} m^{*} k_{\mathrm{f}}^{4}}{(2 \pi)^{3}} \times \frac{2 k_{\mathrm{f}} t \cos 2 k_{\mathrm{f}} t-\sin 2 k_{\mathrm{f}} t}{\left(2 k_{\mathrm{f}} t\right)^{4}} \\
& \times\left(-M_{\mathrm{F}} m_{\mathrm{AF}} t_{\mathrm{F}} \cos \theta\right)
\end{aligned}
$$

is the indirect exchange coupling (RKKY interaction) between the $\overrightarrow{\mathrm{M}}_{\mathrm{F}}$ and $\vec{m}_{\mathrm{AF}}$ at zero temperature [12]. It should be noted that the unit of $\varepsilon_{\text {dip }}$ and $\varepsilon_{\mathrm{RKKY}}$ is energy per unit area. In order to study the influence of temperature dependence in the long-range $H_{\mathrm{E}}$, we let

$m_{\mathrm{AF}}(T)=m_{0}\left(1-b T^{2}\right)$

to $m_{\mathrm{AF}}$ [13], and include Fermi-Dirac distribution in $E_{\mathrm{RKKY}}(t)$

$$
\begin{aligned}
\varepsilon_{\mathrm{RKKY}}(t, T)= & \frac{2 m^{*} J^{2} \overrightarrow{\mathrm{M}}_{\mathrm{F}} \cdot \vec{m}_{\mathrm{AF}}}{(2 \pi)^{6}} t_{\mathrm{F}} \\
& \times \int_{0}^{\infty} \mathrm{d}^{3} k \frac{1}{\mathrm{e}^{\left(\left(\hbar^{2} k^{2} / 2 m\right)-\mu\right) / k_{\mathrm{B}} T}+1} \\
& \times \int_{0}^{\infty} \mathrm{d}^{3} k^{\prime}\left(1-\frac{1}{\mathrm{e}^{\left(\left(\hbar^{2} k^{\prime 2} / 2 m\right)-\mu\right) / k_{\mathrm{B}} T}+1}\right) \\
& \times \frac{\mathrm{e}^{-\mathrm{i}\left(\vec{k}-\vec{k}^{\prime}\right) \cdot \vec{t}}}{k^{2}-k^{\prime 2}} .
\end{aligned}
$$

For the low-temperature limit,

$$
\begin{aligned}
\varepsilon_{\mathrm{RKKY}}(t, T)= & \varepsilon_{\mathrm{RKKY}}(t)+\frac{J^{2} m^{3} k_{\mathrm{B}}^{2}}{24 \pi \hbar^{6} k_{\mathrm{f}}^{2}} \frac{\sin 2 k_{\mathrm{f}} t}{t^{2}} \\
& \times T^{2}\left(-M_{\mathrm{F}} m_{\mathrm{AF}} t_{\mathrm{F}} \cos \theta\right),
\end{aligned}
$$

where $k_{\mathrm{B}}$ is Boltzmann's constant, and $k_{\mathrm{f}}$ is Fermi wave vector. Therefore,

$$
\begin{aligned}
\varepsilon(t, T)= & K_{\mathrm{F}} t_{\mathrm{F}} \sin ^{2} \theta-H M_{\mathrm{F}} t_{\mathrm{F}} \cos \theta \\
& +\varepsilon_{\mathrm{dip}}(t, T)+\varepsilon_{\mathrm{RKKY}}(t, T),
\end{aligned}
$$

and the displacement of hysteresis loop is found to be

$$
\begin{aligned}
H_{\mathrm{E}}= & \frac{\varepsilon_{\mathrm{dip}}(t, T)+\varepsilon_{\mathrm{RKKY}}(t, T)}{M_{\mathrm{F}} t_{\mathrm{F}} \cos \theta} \\
= & {\left[\frac{1}{t^{3}}+\frac{4 J^{2} m^{*} k_{\mathrm{f}}^{4}}{(2 \pi)^{3}}\right.} \\
& \times \frac{2 k_{\mathrm{f}} t \cos 2 k_{\mathrm{f}} t-\sin 2 k_{\mathrm{f}} t}{\left(2 k_{\mathrm{f}} t\right)^{4}} \\
& \left.+\frac{J^{2} m^{3} k_{\mathrm{B}}^{2}}{24 \pi \hbar^{6} k_{\mathrm{f}}^{2}} \frac{\sin 2 k_{\mathrm{f}} t}{t^{2}} T^{2}\right] \\
& \times m_{0}\left(1-b T^{2}\right),
\end{aligned}
$$

where $m_{0}$ and $b$ are our fitting parameter. Fig. 2 exhibits results for Eq. (8) at different temperature. We can find that the RKKY interaction competes with the dipolar interaction, and the oscillatory only becomes significant as the RKKY interaction dominates. At low temperature, the dipolar interaction is stronger than the RKKY interaction, thus the oscillatory behavior is almost suppressed. However the RKKY interaction becomes dominated as temperature increases hence the $H_{\mathrm{E}}$ is oscillatory with the variation of film thickness.

In summary, we have analyzed the long-range oscillation $H_{\mathrm{E}}$ in $\mathrm{FM} / \mathrm{M} / \mathrm{AFM}$ trilayer system. The RKKY interaction and dipolar interaction are used to analyze the temperature effects and thickness dependence of the $H_{\mathrm{E}}$ in $\mathrm{FM} / \mathrm{M} / \mathrm{AFM}$ structures. The results show, at low temperature, the $H_{\mathrm{E}}$ almost decays monotonically with the increasing thickness of metal. When increasing temperature, the $H_{\mathrm{E}}$ becomes oscillatory with thickness of metal.

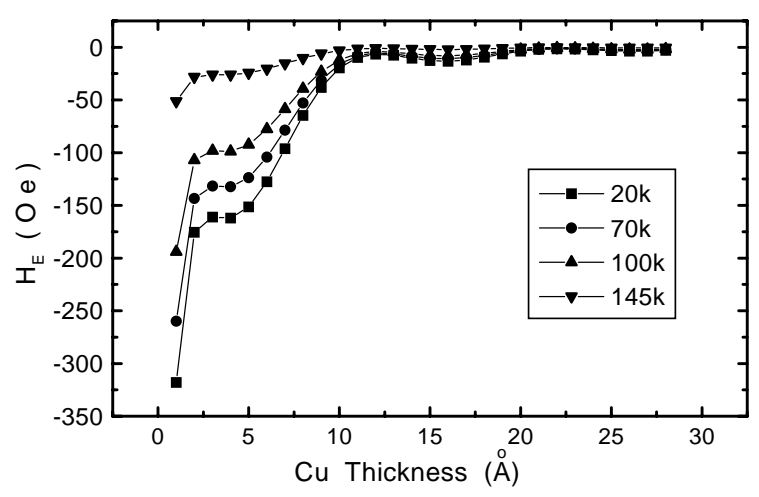

Fig. 2. Temperature dependence of the $H_{\mathrm{E}}$ in FM/M/AFM for the different thickness of the metal spacer. Here $m_{0}$ is $6 \times 10^{-20}$ emu, $b$ is $4 \times 10^{-5} \mathrm{~K}^{-2}$, and $k_{\mathrm{f}}$ is $\pi / 10 \AA^{-1}$. 


\section{References}

[1] W.H. Meiklejohn, C.P. Bean, Phys. Rev. 102 (1956) 1413. [2] W.H. Meiklejohn, C.P. Bean, Phys. Rev. 105 (1957) 904.

[3] S.S.P. Parkin, N. More, K.P. Roche, Phys. Rev. Lett. 64 (1990) 2304.

[4] Y. Wang, P.M. Levy, J.L. Fry, Phys. Rev. Lett. 65 (1990) 2732.

[5] S.S.P. Parkin, R. Bhadra, K.P. Roche, Phys. Rev. Lett. 66 (1991) 2152.

[6] W. Baltensperger, J.S. Helman, Appl. Phys. Lett. 57 (1990) 2954.

[7] P. Bruno, C. Chappert, Phys. Rev. Lett. 67 (1991) 1602.
[8] N.J. Gökemeijer, T. Ambrose, C.L. Chien, Phys. Rev. Lett. 79 (1997) 4270.

[9] L. Thomas, A.J. Kellock, S.S.P. Parkin, J. Appl. Phys. 87 (2000) 5061.

[10] T. Mewes, B.F.P. Roos, S.O. Demokritov, B. Hillebrands, J. Appl. Phys. 87 (2000) 5064.

[11] Minn-Tsong Lin, C.H. Ho, Ching-Ray Chang, Y. D. Yao, Phys. Rev. B 63 (2001) 100404(R).

[12] C. Kittel, Quantum Theory Solids, Wiley, New York, 1963, p. 364 (Chapter 18).

[13] Z.Q. Qiu, J.E. Mattson, C.H. Sowers, U. Welp, S.D. Bader, H. Tang, J.C. Walker, Phys. Rev. B 45 (1992) 2252. 